\title{
The Development of Infective Endocarditis and Oral Dyskinesia in a Type 2 Diabetic Patient
}

\author{
Hiroki Adachi ${ }^{\mathrm{a}}$, Hidekatsu Yanai ${ }^{\mathrm{a}, \mathrm{b}, \mathrm{e}}$, Tetsuo Ichinose ${ }^{\mathrm{c}}$, Yuriko Tanaka ${ }^{\mathrm{c}}$, \\ Hiroyuki Takakura ${ }^{a}$, Kazuhiro Honda ${ }^{\text {b, }}$
}

\begin{abstract}
The group A streptococcus infection has been reported to be associated with the development of neuropsychiatric symptoms in children such as Sydenham's chorea and pediatric autoimmue neuropsychiatric disorders associated with streptococci, possibly due to autoimmunity. However, there are very few literatures discuss the association between neuropsychiatric manifestations and Streptococcus viridians infection, especially in the elderly people. Here, we show a 74-year-old type 2 diabetic woman who developed infective endocarditis infected by Streptococcus viridans and oral dyskinesia.
\end{abstract}

Keywords: Diabetes; Infective endocarditis; Oral dyskinesia; Streptococcus viridans

\section{Introduction}

The association between group A streptococcus (GAS) infection and neuropsychiatric symptoms in children has been reported. Sydenham's chorea is the neurological expression

Manuscript accepted for publication August 23, 2011

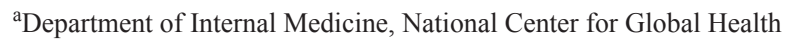
and Medicine, Kohonodai Hospital, Ichikawa, Japan

${ }^{\mathrm{b}}$ Clinical Research Center, National Center for Global Health and Medicine, Kohonodai Hospital, Ichikawa, Japan

${ }^{\mathrm{c}}$ Department of Cardiology, National Center for Global Health and Medicine, Kohonodai Hospital, Ichikawa, Japan

${ }^{\mathrm{d} D e p a r t m e n t}$ of Neurology, National Center for Global Health and Medicine, Kohonodai Hospital, Ichikawa, Japan

${ }^{\mathrm{e}}$ Corresponding author: Hidekatsu Yanai, Department of Internal Medicine, Clinical Research Center, National Center for Global Health and Medicine Kohnodai Hospital, 1-7-1 Kohnodai, Ichikawa, Chiba 272-8516, Japan. Email: dyanai@hospk.ncgm.go.jp

doi:10.4021/jmc286w of acute rheumatic fever, and is characterized by uncoordinated movements, emotional instability, and hypotonia [13]. The pathogenesis of this disease is thought to involve streptococcus-induced autoantibodies which cross-react with antigens of the basal ganglia $[2,4]$. The obsessive-compulsive disorder or tics/Tourette's syndrome in childhood to antecedent GAS, which has been termed pediatric autoimmue neuropsychiatric disorders associated with streptococci (PANDAS), has been also reported [5-7]. Recently, GAS has been receiving attention as a potential cause of neuropsychiatric symptoms, which is possibly due to autoimmunity.

However, to our knowledge, the association between neuropsychiatric manifestations and Streptococcus viridians infection in the elderly people has not ever been described. We will show a 74-year-old type 2 diabetic woman who developed infective endocarditis infected by Streptococcus viridans and oral dyskinesia.

\section{Case Report}

A 74-year-old woman was admitted to our hospital due to the development of oral dyskinesia. At the age of 59 she has been diagnosed as type 2 diabetes. Recent control of blood glucose is good, and plasma glucose level was $187 \mathrm{mg} / \mathrm{dL}$ and hemoglobin A1C level was $6.1 \%$ by dietary treatment. She developed oral dyskinesia 10 years ago, and her oral dyskinesia spontaneously disappeared in two years after the onset. In January 2011, she developed oral dyskinesia after the flu-like symptoms, and her oral dyskinesia disappeared in one month. In March 2011, she again developed oral dyskinesia. On the admission, she presented with oral dyskinesia, and her consciousness was normal. Her artificial teeth did not fit her, and poor oral hygiene was observed. Her body temperature was $36.8{ }^{\circ} \mathrm{C}$ and blood pressure was 161/64 mmHg. Her body weight was $37.4 \mathrm{~kg}$ and height $147 \mathrm{~cm}$ (BMI $17.3 \mathrm{~kg} / \mathrm{m}^{2}$ ). Auscultation revealed a systolic murmur. Laboratory data showed normal leukocyte counts $(6800 / \mu \mathrm{L})$ and slightly elevated serum levels of C-reactive protein $(0.86 \mathrm{mg} / \mathrm{dL}$; normal range, $0-0.3 \mathrm{mg} / \mathrm{dL})$ and brain natriuretic peptide $(134.9 \mathrm{pg} / \mathrm{mL}$; normal range, $0-18.4 \mathrm{pg} /$ $\mathrm{mL})$. 


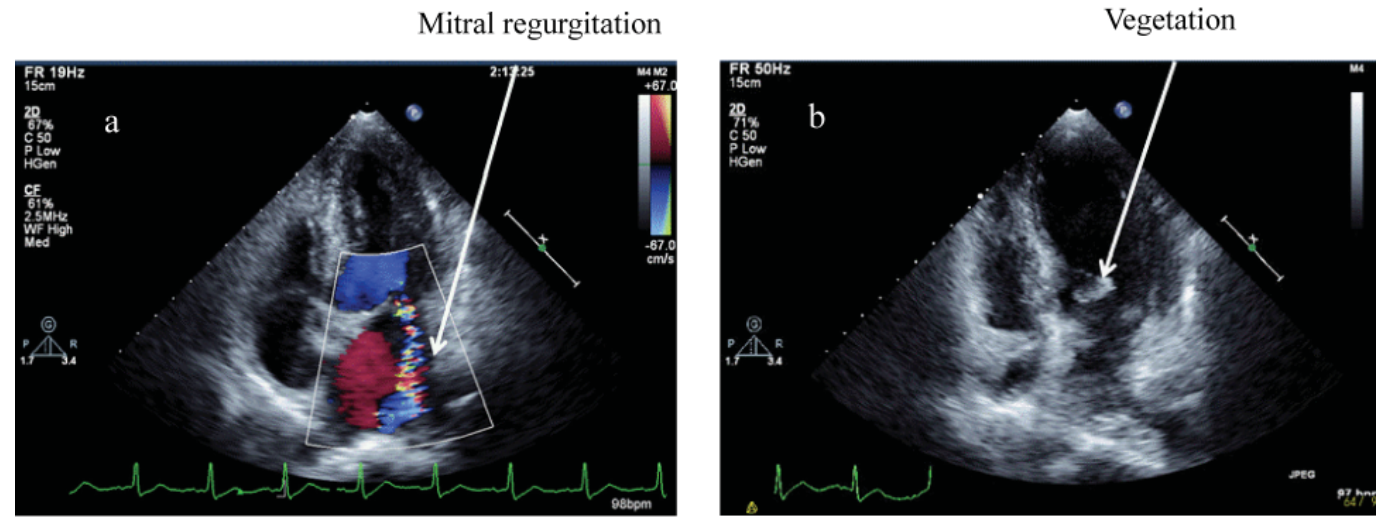

Figure 1. The ultrasound echocardiography revealed mitral regurgitation (a) and vegetation with the diameter of $11.6 \mathrm{~mm}$ at anterior cusp of mitral valve (b).

After the admission, she presented with fever $(>38.5$ oC) at every evening. Cultures of blood grew Streptococcus viridans. The ultrasound echocardiography revealed mitral regurgitation (Fig. 1a) and vegetation with the diameter of $11.6 \mathrm{~mm}$ at anterior cusp of mitral valve (Fig. 1b). She has been finally diagnosed as having infective endocarditis. Magnetic resonance imaging (MRI) did not detect the cause of oral dyskinesia, and somatosensory evoked potentials (SEP) did not show giant SEP, suggesting that her involuntary movement is not due to the damage of cerebral cortex.

\section{Discussion}

The GAS infection has been reported to be associated with the development of neuropsychiatric symptoms in children such as Sydenham's chorea and PANDAS, possibly due to autoimmunity. However, there are very few literatures discuss the association between neuropsychiatric manifestations and Streptococcus viridians infection. In 1989, a 33-year-old woman with an atypical form of amnesia was reported [8]. The amnesia disappeared after the treatment with intravenous penicillin for subacute bacterial endocarditis secondary to Streptococcus viridans infection. However, the precise mechanism has not been elucidated. In 1963, a 40-year-old woman who developed bacterial endocarditis infected by Streptococcus viridans was reported by Medley [9]. She had rapid complex involuntary movements of her arms and legs. Her tongue was tremulous and she showed dysarthria and continuous facial grimacing, which was also observed in our patient. Treatment of penicillin was started and the choreiform movements stopped in 48 hours, and dysarthria and involuntary movements disappeared in the patient by Medley. However, she died three weeks after admission due to acute pulmonary edema. Post-mortem findings revealed vegetations along aortic valve cusps. The brain looked completely normal, and no vascular occlusion could be seen. The reason for chorea in this case could not be determined.
In our case, oral dyskinesia was always bilateral, suggesting an improbable history for embolic episodes, which is supported by the MRI findings. The treatment using antibiotics did not affect oral dyskinesia in our case. She developed oral dyskinesia 10 years ago, and her oral dyskinesia spontaneously disappeared in two years. In January 2011, she developed oral dyskinesia after the flu-like symptoms, and her oral dyskinesia disappeared in one month. In March 2011, she again developed oral dyskinesia. This clinical course suggest that she is prone to develop oral dyskinesia, and that infection including viral or bacterial (Streptococcus viridans) may be associated with the development of oral dyskinesia. Brain MRI and SEP results denied mycotic aneurysm, cerebral infarction, and the damage of cerebral cortex. Infection-induced autoimmunity which is observed in the GAS infection $[2,4]$, may be associated with the development of oral dyskinesia in our case. Conversely, oral dyskinesia, the existence of mitral regurgitation, unfitting artificial teeth and poor oral hygiene may be associated with the development of infective endocarditis. Recent reports suggested that minor gingival trauma such as toothbrushing induces bacteremia, and that poor oral hygiene is a risk factor for infective endocarditis-related bacteremia, supporting our hypothesis $[10,11]$.

In conclusion, it remains unknown whether Streptococcus viridans infection induced oral dyskinesia and how oral dyskinesia induced infective endocarditis, which should be elucidated in the future. To our knowledge, our patient is very rare elderly case who developed infective endocarditis infected by Streptococcus viridans and oral dyskinesia.

\section{References}

1. Mink J, Kurlan R. Acute postinfectious movement and psychiatric disorders in children and adolescents. J Child Neurol. 2011;26(2):214-217.

2. Cardoso F. Sydenham's chorea. Handb Clin Neurol. 
2011;100:221-229.

3. Oosterveer DM, Overweg-Plandsoen WC, Roos RA. Sydenham's chorea: a practical overview of the current literature. Pediatr Neurol. 2010;43(1):1-6.

4. Brilot F, Merheb V, Ding A, Murphy T, Dale RC. Antibody binding to neuronal surface in Sydenham chorea, but not in PANDAS or Tourette syndrome. Neurology. 2011;76(17):1508-1513.

5. Gabbay V, Coffey BJ, Babb JS, Meyer L, Wachtel C, Anam S, Rabinovitz B. Pediatric autoimmune neuropsychiatric disorders associated with streptococcus: comparison of diagnosis and treatment in the community and at a specialty clinic. Pediatrics. 2008;122(2):273-278.

6. Shulman ST. Pediatric autoimmune neuropsychiatric disorders associated with streptococci (PANDAS): update. Curr Opin Pediatr. 2009;21(1):127-130.

7. Murphy TK, Kurlan R, Leckman J. The immunobiol- ogy of Tourette's disorder, pediatric autoimmune neuropsychiatric disorders associated with Streptococcus, and related disorders: a way forward. J Child Adolesc Psychopharmacol. 2010;20(4):317-331.

8. Grillo RA, Jr., Olson NH. Amnesia as a presenting symptom in subacute bacterial endocarditis. J Clin Psychiatry. 1986;47(7):383-384.

9. Medley DR. Chorea and bacterial endocarditis. Br Med J. 1963;1(5334):861-862.

10. Lucas VS, Gafan G, Dewhurst S, Roberts GJ. Prevalence, intensity and nature of bacteraemia after toothbrushing. J Dent. 2008;36(7):481-487.

11. Lockhart PB, Brennan MT, Thornhill M, Michalowicz BS, Noll J, Bahrani-Mougeot FK, Sasser HC. Poor oral hygiene as a risk factor for infective endocarditis-related bacteremia. J Am Dent Assoc. 2009;140(10):1238-1244. 